\title{
Microbial fuel cell fed by Barnett Shale produced water: Power production by hypersaline autochthonous bacteria and coupling to a desalination unit
}

\author{
Oihane Monzon, Yu Yang, Jun Kim, Amy Heldenbrand, Qilin Li, Pedro J.J. Alvarez* \\ Department of Civil E' Environmental Engineering, Rice University, Houston, TX 77005, USA
}

\section{A R T I C L E I N F O}

\section{Article history:}

Received 31 May 2016

Received in revised form

21 September 2016

Accepted 22 September 2016

Available online 26 September 2016

\section{Keywords:}

Microbial fuel cell

Produced water

High salinity

Desalination

H. praevalens

M. hydrocarbonoclasticus

\begin{abstract}
A B S T R A C T
Hydraulic fracturing for oil and gas production can generate large volumes of highly saline produced waters that pose a significant disposal challenge and a potential opportunity for reuse. Here, we report on a microbial fuel cell (MFC) fed with produced water (Barnett Shale), which produced power $\left(47 \mathrm{~mW} / \mathrm{m}^{2}\right)$ and accomplished a COD removal efficiency of $68 \%$ (influent COD 10,520 $\pm 1340 \mathrm{mg} / \mathrm{L}$ ). Bacterial population analysis showed two autochthonous halophilic species colonizing the anode, $H$. praevalens and $M$. hydrocarbonoclasticus. In addition, we demonstrated that hypersaline MFCs $(100 \mathrm{~g} / \mathrm{L} \mathrm{NaCl})$ can produce electricity to power a capacitive deionization (CDI) device for desalination, with a salt adsorption capacity of up to $40 \mathrm{mg}$ of salt per $\mathrm{g}$ of carbon electrode. This is the first report of a MFC operating with hypersaline produced water, and illustrates the potential to couple MFCs with CDI to enable desalination and reuse of hypersaline wastewaters.
\end{abstract}

(c) 2016 Published by Elsevier B.V.

\section{Introduction}

Hydrocarbon production inherently involves the use of relatively large volumes of water, especially for unconventional oil and gas production from shale formations (hydraulic fracturing), which requires about $30,000 \mathrm{~m}^{3}$ per well [1] for oil/gas extraction process. Approximately $30 \%$ of this water flows back as produced or flowback water, which may contain very high salt concentrations (up to $250 \mathrm{~g} / \mathrm{L} \mathrm{NaCl}$ ) as well as a wide variety of pollutants such as hydrocarbons, naturally occurring radioactive materials (NORM), and residual chemicals and biocides used in the hydraulic fracturing fluids [2]. Water management is thus a major challenge for unconventional oil and gas industry, representing about $10 \%$ of the total cost of well operation [3].

Produced waters need to be adequately treated to enable reuse or beneficial disposition. Oil and suspended solids removal is highly efficient with currently available technologies [2]. However, there is a need to remove dissolved organic pollutants, many of which are toxic (e.g., aromatic hydrocarbons) [4]. The high salinity frequently encountered in produced waters is particularly challenging for biological treatment processes, which are usually the more eco-

\footnotetext{
* Corresponding author.

E-mail addresses: alvarez@rice.edu, oihanemonzon@hotmail.com (P.J.J. Alvarez).
}

nomical approach to degrade dissolved organic pollutants but are significantly inhibited by hypersaline conditions that exert osmotic stress and plasmolysis in bacterial cells [5,6].

Desalination of flowback/produced wastewaters may also be required as the salinity in treated water must be below an established limit for surface discharge, such as $500 \mathrm{ppm}$ (i.e., the secondary drinking water standard) in the case of Pennsylvania [3]. In addition, for reuse in subsequent hydraulic fracturing processes, removal of some multivalent cations such as calcium, barium and strontium is also necessary to avoid interference with chemical additives used in the fracturing fluid as well as clogging of the formation and pipelines due to mineral precipitation [7]. Currently, desalination of flowback and produced waters relies on energy-intensive processes such as reverse osmosis or mechanical vapor compression [2]; therefore, there is an emerging need for cost-effective low-energy treatment alternatives for both organic pollutants removal and low-energy desalination.

Microbial fuel cells (MFC) can offer biological treatment of organic pollutants from saline wastewaters while producing electricity, thus representing a great opportunity to mitigate environmental impacts and recover resources in a cost-effective manner. MFCs produce electricity while treating wastewater through bacteria that are able to oxidize organic compounds (i.e., electron donors) under anaerobic conditions while using a solid terminal electron acceptor in the process (e.g., carbon electrodes) [8]. For this specific application bacteria must meet two 
main requirements: thrive under extreme saline conditions and have exoelectrogenic [9] capabilities. Although the use of MFCs for hypersaline wastewaters is almost inexistent and there is no precedent studies of produced water fed MFCs, we previously demonstrated that MFCs can work under extreme saline conditions of $100 \mathrm{~g} / \mathrm{L} \mathrm{NaCl}$ with the appropriate exoelectrogenic halophiles colonizing the anode $[10,11]$. Species belonging to Halanaerobium genus, which have also been found in shale formations and oil reservoirs [12], were found to be predominant in the anode.

Electrical power production by MFCs could be used to power a desalination device, such as capacitive deionization (CDI) that uses charged electrodes to adsorb oppositely charged salt ions [13]. The advantage of using a CDI is that it provides high water recovery and is a low-energy desalination technology with much lower energy requirements than the more commonly-used energy-intensive membrane based desalination methods (i.e., nanofiltration and RO), which are not applicable for salt concentrations higher than $10 \mathrm{~g} / \mathrm{L}$ [14].

MFC-CDI coupling could allow removal of both organic contaminants and salts, requiring minimum external energy supply (if not completely self-powered), which would be a great advantage for the unconventional oil and gas industry that often takes place in off-grid remote sites. Previous studies have combined MFCs with desalination devices [15-17], but never before with a hypersaline MFC. A widely used approach found in literature integrates the MFC and the $\mathrm{CDI}$ in a single device, microbial capacitive desalination cells (MCDC), which have the main drawback that $\mathrm{pH}$ decreases in the anode chamber (inhibiting bacteria and MFC performance) since protons released during degradation of organic compounds cannot travel to the cathode to recombine with $\mathrm{O}_{2}$ and electrons to form water [17].

No previous publications have tested the possibility of MFCs to treat hypersaline produced waters and use the generated electricity to power a desalination device. In this paper we assess whether an MFC can perform when fed directly with produced wastewater with autochthonous bacteria from shale gas/oil formation. Proof of concept that hypersaline MFCs $(100 \mathrm{~g} / \mathrm{L} \mathrm{NaCl})$ can generate enough electricity to drive desalination in a separate device (i.e., a commercial CDI unit) is also presented.

\section{Materials and methods}

\subsection{MFC reactor}

A single chamber batch-fed air cathode MFC was used to assess the viability of exoelectrogenic bacteria in real produced wastewater. Materials and experimental set up were previously described [10].

The produced wastewater used in this study was collected from Barnett Shale formation (Bend Arch-Fort Worth Basin Province, TX) from a well that had been operating for 100 days. Some characteristics from these sample include a turbidity of $178 \mathrm{NTU}, \mathrm{pH}=6.9$, Total dissolved solids $($ TDS $)=85.7 \mathrm{~g} / \mathrm{L}$ and $\mathrm{COD}=10.52 \pm 1.34 \mathrm{~g} / \mathrm{L}$. The MFC was directly fed with produced wastewater as the only source of bacteria and nutrients, and it was monitored by voltage production through a multimeter (model 2100, Keithley Instruments Inc, Cleveland, OH) with an external load of $510 \Omega$. COD degradation was measured using a HACH HR COD colorimetric assay (Hach Company, CO). Serial dilutions were made until the $\mathrm{Cl}^{-}$ concentration was within the range recommended by the manufacturer (upper limit $1000 \mathrm{mg} / \mathrm{L} \mathrm{Cl}^{-}$for diluted samples).

\subsection{Analysis of microbial population evolution in the MFC}

Pyrosequencing analysis was performed after three months of MFC operation. The available sample volume of Barnett produced water was limited $(\sim 0.5 \mathrm{~L})$ and allowed only for the acclimation and first feed of the MFC. This was enough to allow the bacteria from produced wastewater to colonize the anode and generate electricity. In the subsequent cycles synthetic hypersaline media was used as replacement; which contained sodium benzoate $(1 \mathrm{~g} / \mathrm{L})$, $\mathrm{MgCl}_{2} .6 \mathrm{H}_{2} \mathrm{O}(30 \mathrm{~g} / \mathrm{L}), \mathrm{KCl}(7 \mathrm{~g} / \mathrm{L})$, Yeast $(8.5 \mathrm{~g} / \mathrm{L})$, peptone $(1.7 \mathrm{~g} / \mathrm{L})$, casamino acids $(1.7 \mathrm{~g} / \mathrm{L})$ and $100 \mathrm{~g} / \mathrm{L} \mathrm{NaCl}$. The $\mathrm{pH}$ was adjusted to 7.5. The original produced water from Barnett Shale, the bacteria colonizing the anode and bacteria from the bulk fluid of the MFC were analyzed by pyrosequencing following the method previously described [10].

\subsection{Coupling of the MFCs with capacitive deionization device}

The coupling of hypersaline MFCs with a CDI device was performed to evaluate the ability of the electrical power produced by the hypersaline MFCs to drive desalination in the CDI. The CDI device consists of two acrylic sheets with two activated carbon electrodes (Material Methods PACMM 203, Irvine, CA) squeezed in between, separated by filter paper (Whatman Qualitative Filter Paper, 20-25 $\mu \mathrm{m}$, Little Chalfont, UK). Leaking was prevented by fitting an O-ring (433 N70 Buna-N Nitrile 70 O-ring, Lwiston, ID) in a groove placed into the acrylic frame. Water circulated using a peristaltic pump (SciLog Tandem 1081) and tubing (Masterflex Tubing PuriFlex, L/S 13, Vernon Hills, IL). To measure conductivity changes in the system, a microconductimeter fitted to a conductivity probe was employed (Oakton 4-cell conductivity/temperature probe, PC 2700 Benchtop meter).Current was monitored by placing a multimeter (Extech EX540, True RMS Industrial Multimeter/Datalogger, Nashua, $\mathrm{NH}$ ) in series with the CDI device.

As proof of concept the coupling was made using three hypersaline MFCs fed with bacteria from Galveston bay pond connected in parallel to supply the CDI. MFCs for the CDI-coupling experiments were not fed with the same produced water used to develop the initial MFC reactor due to the limited amount of produced water sample. Nevertheless, note that the culture from Galveston bay was an enrichment ( $\sim 90 \%)$ of Halanaerobium species [10], which are also predominant species in the original MFC fed with produced water. With parallel configuration the current of each MFC sums up. The influent of saline water into the CDI contained $0.5 \mathrm{~g} / \mathrm{L} \mathrm{NaCl}$, and the flow rate was of $0.1 \mathrm{~mL} / \mathrm{min}$, to allow sufficient time for the desalination to take place.

\section{Results and discussion}

\subsection{MFC operation with real produced wastewaters}

Produced wastewater from Barnett Shale formation that was collected from a well after 100 days of production was used as the only source of bacteria and electron donors in the MFC. Fig. 1 shows the power density and polarization curves of the MFC only fed with produced water (TDS $=86,000 \mathrm{mg} / \mathrm{L}$ ). Bacteria from the Barnett Shale colonized the MFC anode and transferred electrons to its surface to produce a maximum power density of $47 \mathrm{~mW} / \mathrm{m}^{2}(0.14 \mathrm{~V}$ and $0.42 \mathrm{~mA})$, comparable to the power density of $71 \mathrm{~mW} / \mathrm{m}^{2}$ obtained using a mixed culture from Galveston Bay under $100,000 \mathrm{mg} / \mathrm{L} \mathrm{NaCL}$ [10]. The COD removal efficiency was of $68 \%\left(\mathrm{COD}_{\text {out }}=3350 \pm 70 \mathrm{mg} / \mathrm{L}\right)$, yielding a Coulombic efficiency of $10 \%$ over 58 days.

Given the limited quantity of produced water sample available, the MFC was fed with a synthetic saline growth medium in sub- 


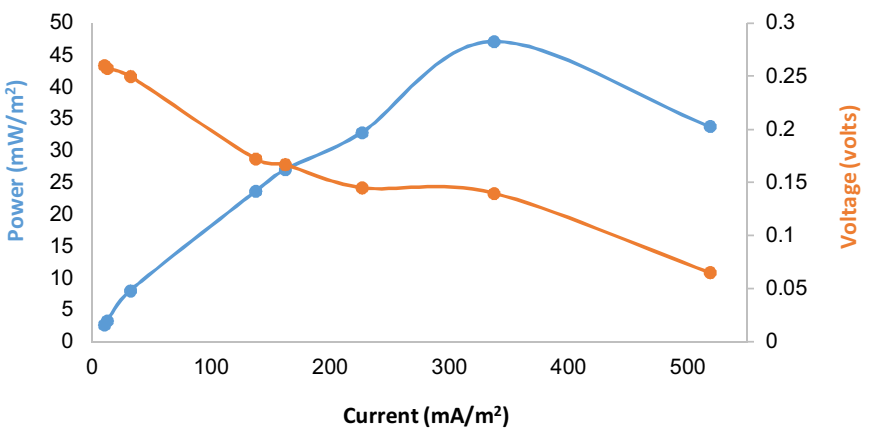

Fig. 1. Polarization and power density curves of produced wastewater fed MFC;. External load ranges from 100 to $20,000 \Omega$.

sequent cycles of the experiment, containing $100,000 \mathrm{mg} / \mathrm{L} \mathrm{NaCl}$. Mean values of the maximum steady voltage produced in three subsequent cycles with autochthonous bacteria from shale and synthetic saline media were of $0.155 \pm 0.023 \mathrm{~V}$ with an external load of $510 \Omega$.

These results demonstrate that MFC can be operated with real produced wastewater as the only feed stream without additional bacterial seeding, and represent a step towards field application.

\subsection{Phylogenetic analysis of bacteria in the MFC fed with real produced wastewater}

Pyrosequencing results of bacterial population in Barnett Shale produced wastewater, as well as of bacteria colonizing the anode and bulk fluid of the MFC initially fed with Barnett Shale produced wastewater after 3 months of continuous operation are shown in Fig. 2.

Bacterial population in produced wastewater significantly differs from that found in the MFC. $H$. praevalens, which was not detected in Barnett Shale sample probably due to its relatively small population $(<1 \%)$, became the predominating species in the MFC. This shift can be attributed to the fact that after feeding the MFC with real produced wastewater subsequent medium replacements were done with synthetic media, which apparently favors the growth of $H$. praevalens as observed in a different hypersaline MFC fed with bacteria from saline pond [10]. Interestingly, Marinobacter hydrocarbonoclasticus, which was present in the original Barnett Shale in large numbers, dominated also the population on the MFC anode surface as well as in bulk fluid. Marinobacter hydrocarbonoclasticus is of great interest due to its extraordinary ability to degrade petroleum hydrocarbons (typically non-cyclic alkanes) and its capacity to use them as the sole source of energy and carbon. It degrades aromatic compounds under anaerobic conditions and is thus important in the field of bioremediation [18]. In addition, $M$. hydrocarbonoclasticus has unique features such as extreme salt tolerance which ranges from 5000 to $205,000 \mathrm{mg} / \mathrm{L} \mathrm{NaCl}$, and the capacity to grow either in aerobic or anaerobic conditions, which provides a competitive advantage. $M$. hydrocarbonoclasticus was first isolated in Mediterranean Sea nearby a petroleum refinery [19]. It is described as Gram (-), aerobic and rod-shaped bacteria, and its genome of 4 million base-pares size was sequenced in 2012 [20].

The ease of $M$. hydrocarbonoclasticus to form biofilms at the interface of water and hydrophobic hydrocarbons has been demonstrated [20], which is consistent with our observation of this species becoming dominant in the carbon brush anode of the hypersaline MFC. Although its presence in the bulk fluid is also high, the species abundance percentages are inverted in the anode with respect to the bulk fluid as M. hydrocarbonoclasticus has greater capacity to form biofilm than $H$. praevalens [10]. The presence of $M$. hydrocarbonoclasticus in electroactive biofilms has also been reported,
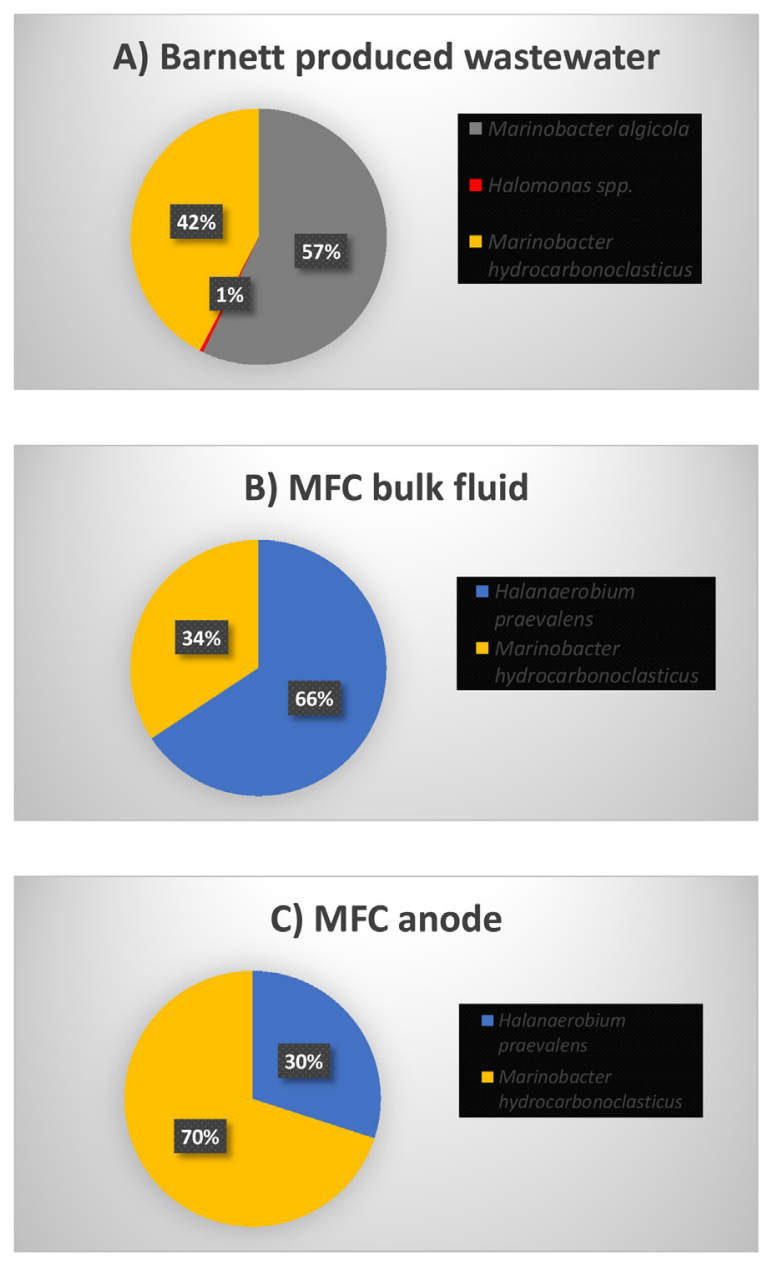

Fig. 2. Predominant bacterial species in (A) Barnett Shale produced water, (B) MFC bulk fluid and (C) on the MFC anode inoculated with Barnett Shale.

specifically in marine electroactive biocathodes for biosensor applications [21]. Its capacity to extracellularly transfer electrons has been evidenced when producing current as a single species in a biocathode [22].

These features of Marinobacter hydrocarbonoclasticus are highly beneficial for MFCs aimed at organic degradation and electricity generation under extreme saline conditions. For example, the presence of $M$. hydrocarbonoclasticus may explain the higher COD removal efficiency of this MFC (68\%) compared to another hypersaline MFC populated primarily by Halanaerobium species (20\%) [10]. This suggests the possibility that M. hydrocarbonoclasticus may hydrolyze complex hydrocarbons and convert them to substrates that could be fermented by other organisms (e.g., Halanaerobium 
spp.), enabling potential commensalistic relationships that benefit overall MFC performance. The microbial analysis of the MFC, both of bulk fluid and anode, shows that $M$. hydrocarbonoclasticus and $H$. praevalens, both autochthonous bacteria from the Barnett shale formation, can coexist in an electroactive biofilm of an hypersaline MFC and contribute to electrical power production. This highlights that MFCs have the potential to be fed and perform with bacteria from real produced wastewaters, demonstrating the potential feasibility of the hypersaline MFC for oil and gas produced wastewater treatment.

\subsection{Coupling of hypersaline MFCs with CDI}

With the electrical output produced by hypersaline MFCs, we attempted to directly power a CDI device to drive desalination. The electric power produced by the MFCs generated a potential difference between the CDI electrodes, oppositely charged and so, with the ability to adsorb saline counter ions on their surface. When coupling MFCs with a CDI there is no longer a constant external load $\left(R_{\text {ext }} \neq 510 \Omega \neq\right.$ Const.) applied to the MFCs. The external resistor of the MFC is now the CDI device, which behaves as a capacitor whose resistance increases continuously as the electrodes are charged and desalination takes place. As charging of the CDI electrodes proceeds, the resistance of the CDI device increases, leading to increased voltage output of the MFC. The voltage $(\mathrm{V})$ generated by the MFC influences the total number of charges that the CDI electrodes can store and hence the number of ions the CDI is able to remove from water (adsorption capacity), while the produced current (I) is related to the rate at which the CDI electrodes are charged and therefore it determines the ion adsorption kinetics. Through Ohms Law $\left(\mathrm{V}=\mathrm{I}^{*} \mathrm{R}\right)$ we can predict that as desalination progresses, the external resistance in the MFC will increase, producing an increase of the voltage output, while sacrificing current. The electric power produced by the MFC will generate a potential difference between the CDI electrodes that will be oppositely charged and so adsorbing saline counter ions on their surface.

Three hypersaline MFCs where connected in parallel as shown in Fig. 3. The voltage produced by the MFCs was in the range of $0.17-0.21 \mathrm{~V}$ with $R_{\text {ext }}=510 \Omega$ prior to the coupling with the CDI.

The evolution of the voltage applied to the CDI with respect to time is shown in Fig. 4 for two subsequent desalination cycles without media replacement of the MFCs. Initially, electrodes had zero electric potential (uncharged) and as empowered by MFCs, continuously generating current, a charge difference between the two electrodes from the CDI is produced. Voltage reading showed sharp increase from the initial point and stabilizing at about $0.4 \mathrm{~V}$.

The increasing applied voltage to CDI electrodes determined the tendency of salt adsorption (Fig. 5). Two different cycles at same flow rate show similar adsorption trends. The adsorption rate at maximum salt removal was $0.53-0.59 \mu \mathrm{g} / \mathrm{min}$ and due to the higher current (sum up of the three MFCs) the maximum adsorption rate was reached in a short period of time. The salt adsorption density up to $41.6 \mathrm{~h}(2500 \mathrm{~min})$ was in the range of $0.30-0.40 \mathrm{mg} / \mathrm{g}$ ( $\mathrm{mg}$ of salt per $\mathrm{g}$ of carbon electrode). Although optimization is still needed, these results demonstrate that the energy produced by a hypersaline MFC can drive desalination on a CDI, making plausible the use of the hybrid MFC-CDI system for treatment of saline flowback and produced waters from shale gas and oil production. The overall adsorption capacity was limited by the surface area and charge density of the commercial CDI electrodes. Research in electrode materials such as nanotechnology-enhanced electrodes that exhibit higher surface area and adsorption capacity will offer an opportunity to improve the CDI desalination process [23,24].

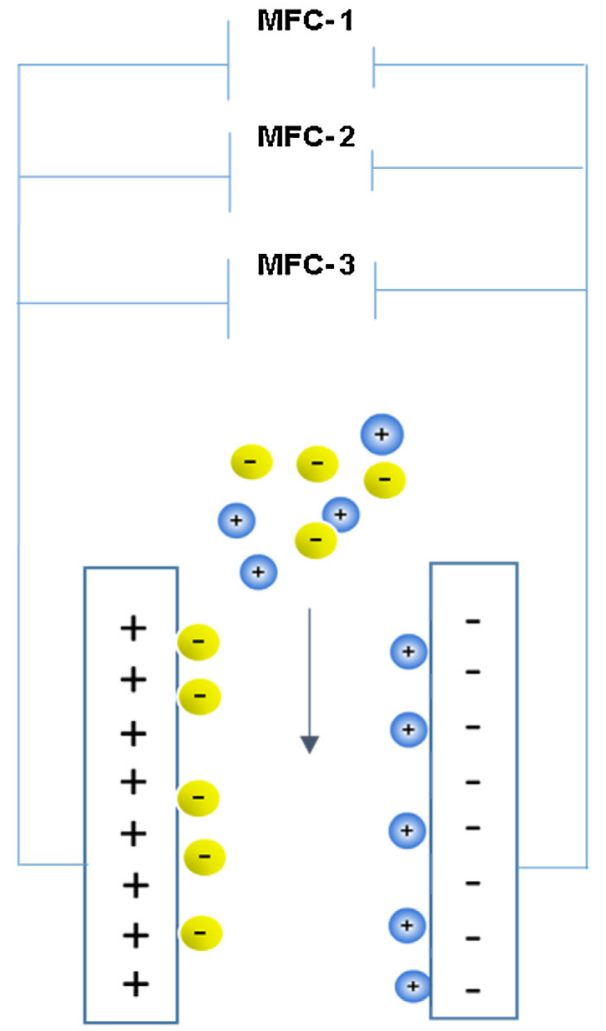

Fig. 3. Scheme of three MFCs in parallel powering the CDI.

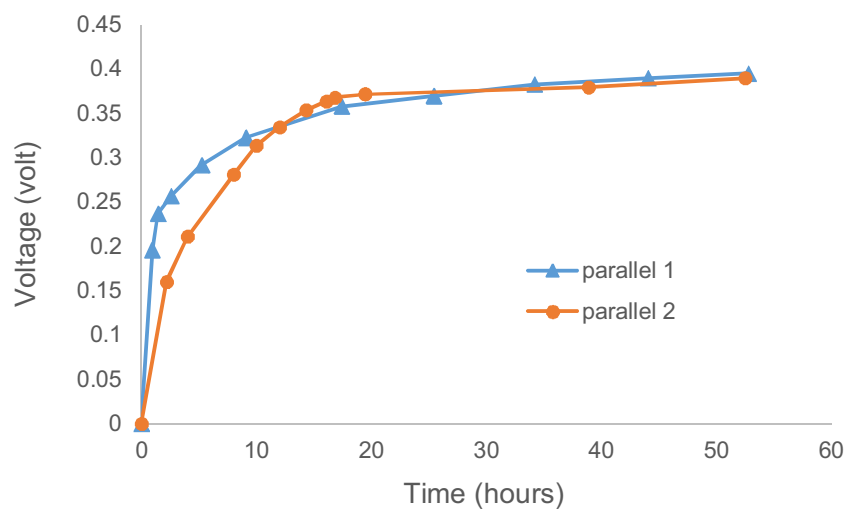

Fig. 4. Voltage evolution in the CDI in two different experiments (parallel 1 and parallel 2) produced by three hypersaline MFCs connected in parallel.

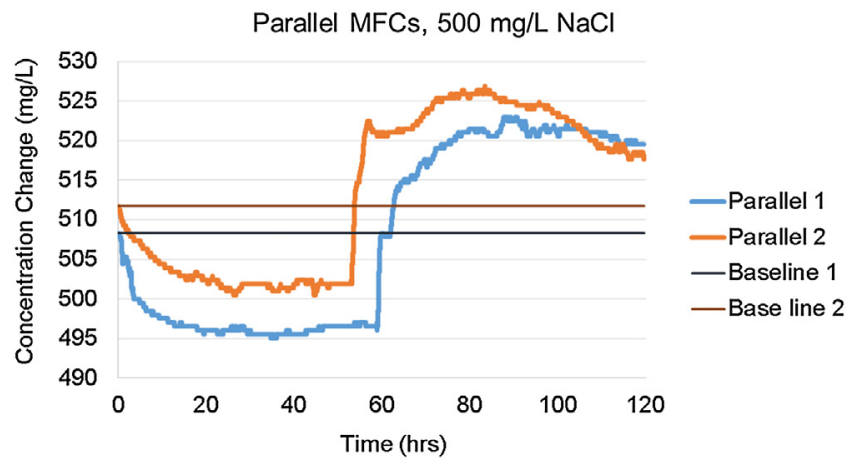

Fig. 5. Salt removal in the CDI ( 2 cycles) fed with 3 parallel-MFCs. 


\section{Conclusions}

This study demonstrates that a hypersaline MFC can be operated with real produced water as the only feed stream without additional bacterial seeding.

A new bacterium indigenous to the Barnett shale formation, Marinobacter hydrocarbonoclasticus, was found to co-colonize the anode with $H$. praevalens in a MFC that produces a sustained power output and accomplished significant COD removal efficiency (68\%) during several months of continuous operation. Marinobacter hydrocarbonoclasticus offers several advantages for hypersaline MFCs due to their proven exoelectrogenic activity, extreme saline tolerance and petroleum hydrocarbon degradation capability.

Although optimization is still needed, the MFC-CDI coupling results demonstrate that the energy produced by a hypersaline MFC can drive desalination in a CDI unit, making plausible the use of an hybrid MFC-CDI system for treatment of saline flowback and produced waters from shale gas and oil production.

\section{Acknowledgement}

This research work was supported by BP North America.

\section{References}

[1] M. Jiang, C.T. Hendrickson, J.M. VanBriesen, Life cycle water consumption and wastewater generation impacts of a Marcellus Shale gas well, Environ. Sci. Technol. 48 (3) (2014) 1911-1920.

[2] F.R. Ahmadun, et al., Review of technologies for oil and gas produced water treatment, J. Hazard. Mater. 170 (2-3) (2009) 530-551.

[3] B.G. Rahm, et al., Wastewater management and Marcellus Shale gas development: trends, drivers, and planning implications, J. Environ. Manage. 120 (2013) 105-113.

[4] K.B. Gregory, R.D. Vidic, D.A. Dzombak, Water management challenges associated with the production of shale gas by hydraulic fracturing, Elements 7 (3) (2011) 181-186.

[5] B.M. Peyton, T. Wilson, D.R. Yonge, Kinetics of phenol biodegradation in high salt solutions, Water Res. 36 (19) (2002) 4811-4820.

[6] X. Shi, et al., Sequential anaerobic-aerobic treatment of pharmaceutical wastewater with high salinity, Bioresour. Technol. 153 (2014) 79-86.
[7] O. Olsson, D. Weichgrebe, K.-H. Rosenwinkel, Hydraulic fracturing wastewater in Germany: composition, treatment, concerns, Environ. Earth Sci. 70 (8) (2013) 3895-3906

[8] H.-S. Lee, et al., Evaluation of energy-conversion efficiencies in microbial fuel cells (MFCs) utilizing fermentable and non-fermentable substrates, Water Res. 42 (6-7) (2008) 1501-1510.

[9] B.E. Logan, J.M. Regan, Electricity-producing bacterial communities in microbial fuel cells, Trends Microbiol. 14 (12) (2006) 512-518.

[10] O. Monzon, et al., Microbial fuel cells under extreme salinity: performance and microbial analysis, Environ. Chem. 12 (3) (2014) 293-299 (2015).

[11] O. Monzon, et al., Quorum sensing autoinducers enhance biofilm formation and power production in a hypersaline microbial fuel cell, Biochem. Eng. J. 109 (2016) 222-227.

[12] J.P. Davis, C.G. Struchtemeyer, M.S. Elshahed, Bacterial communities associated with production facilities of two newly drilled thermogenic natural gas wells in the barnett shale (Texas, USA), Microb. Ecol. 64 (4) (2012) 942-954.

[13] Y. Oren, Capacitive deionization (CDI) for desalination and water treatment-past, present and future (a review), Desalination 228 (1-3) (2008) 10-29.

[14] R. Zhao, et al., Energy consumption in membrane capacitive deionization for different water recoveries and flow rates, and comparison with reverse osmosis, Desalination 330 (2013) 35-41.

[15] C. Forrestal, et al., Microbial desalination cell with capacitive adsorption for ion migration control, Bioresour. Technol. 120 (2012) 332-336.

[16] Y. Kim, B.E. Logan, Microbial desalination cells for energy production and desalination, Desalination 308 (2013) 122-130.

[17] M. Mehanna, et al., Using microbial desalination cells to reduce water salinity prior to reverse osmosis, Energy Environ. Sci. 3 (8) (2010) 1114-1120.

[18] M. Shahriari Moghadam, et al., Biodegradation of polycyclic aromatic hydrocarbons by a bacterial consortium enriched from mangrove sediments, J. Environ. Health Sci. Eng. 12 (1) (2014) 014-0114.

[19] B. Klein, et al., Cytoplasmic wax ester accumulation during biofilm-driven substrate assimilation at the alkane-water interface by Marinobacter hydrocarbonoclasticus SP17, Res. Microbiol. 159 (2) (2008) 137-144.

[20] R. Grimaud, et al., Genome sequence of the marine bacterium Marinobacter hydrocarbonoclasticus SP17, which forms biofilms on hydrophobic organic compounds, J. Bacteriol. 194 (13) (2012) 3539-3540.

[21] I. Vandecandelaere, et al., Bacterial diversity of the cultivable fraction of a marine electroactive biofilm, Bioelectrochemistry 78 (1) (2010) 62-66.

[22] S.M. Strycharz-Glaven, et al., Electrochemical investigation of a microbial solar cell reveals a nonphotosynthetic biocathode catalyst, Appl. Environ. Microbiol. 79 (13) (2013) 3933-3942.

[23] H. Li, et al., Novel graphene-like electrodes for capacitive deionization, Environ. Sci. Technol. 44 (22) (2010) 8692-8697.

[24] T. Humplik, et al., Nanostructured materials for water desalination, Nanotechnology 22 (29) (2011) 0957-4484. 